\title{
Performance of Transmit Antenna Selection in Multiple Input Multiple Output-Orthogonal Space Time Block Code (MIMO- OSTBC) System Joint with Bose-Chaudhuri-Hocquenghem (BCH)-Turbo Code (TC) in Rayleigh Fading Channel
}

\author{
Mr. Nitin Tejram Deotale ${ }^{1^{*}}$ \\ ${ }^{1}$ Lokmanya Tilak College of Engineering, Navi Mumbai, Maharashtra, INDIA \\ *Corresponding Author
}

DOI: https://doi.org/10.30880/ijie.2021.13.06.018

Received 17 Feburary 2020; Accepted 13 December 2020; Available 31 August 2021

\begin{abstract}
The Transmit antenna selection (TAS) technique is essential to increase the efficiency of spatial modulation (SM) systems. This TAS is an effective technique for reducing the Multiple Input Multiple Output (MIMO) systems, which can increase the computational difficulty and Bit error rate (BER) by various TAS algorithms. But these selection methods cannot provide code gain, so it is essential to join the external code in TAS to obtain code gain advantages in BER. In some existing work, the improved BER has been perceived by joining Forward Error Correction Code (FEC) and Space Time Block Code (STBC) for MIMO systems provided greater code gain. A multiple TAS-OSTBC technique with new integration of Bose-Chaudhuri-Hocquenghem (BCH)Turbo code (TC) is proposed in this paper. The TAS-OSTBC system connects with the external BCH code in sequence of internal Turbo code. This combination can provide increasing code gain and the effective advantages of the TAS-OSTBC system. To perform the system analysis Rayleigh channel is utilized. In the case with multiple TAS-OSTBC systems, better performance can provide by this new combination of the BCH-Turbo compared to the conventional Turbo code for the Rayleigh fading.
\end{abstract}

Keywords: Orthogonal Space Time Block Codes (OSTBC),Bit Error Rate (BER), Forward Error Correction Codes (FEC), Transmit Antenna Selection (TAS), Bose-Chaudhuri-Hocquenghem (BCH) codes, Turbo Codes (TC)

\section{Introduction}

In wireless communications, MIMO is an antenna technology that enhances the capacity of the transmission by multiple antennas on the transmitter and receiver side. At every node of the communications circuit the antennas are used to combine reduce errors and improve data speed [1]. MIMO systems can provide a remarkable efficiency gains on conventional single input single-output (SISO) systems. It satisfies to large data rates that require an effective and reliable digital transmission [2]. Multiple RF chains are required for multiple antennas with amplifiers, analog to digital converters, mixers, etc., but they are more expensive [3, 4]. In MIMO system has two techniques, one kind of technique is spatial multiplexing and another kind of technique is Space Time Coding (STC). This may be executed for supplying higher data rates or supplying capacity benefits [5, 6]. MIMO systems use STBC to achieve the maximum diversity gain. To provide the highest throughput by STBC using simple decoding algorithms based on linear processing techniques [7].

To address the complexity of hardware problem of MIMO system the Antenna Selection (AS) system is presented [8]. The subset of antennas are used in AS, which receives according to the channel level data of the system, where the number of receiving antennas is less than or equal to the number of selected transmitter [9]. TAS plays a significant role in selecting the best antenna for transmission subset according to the channel state information feedback which is obtained 
from the receiver. For a transmission purpose in TAS-MIMO system, a single subset of antenna is selected with Maximal Ratio Combining (MRC) [10, 11]. In TAS system for multiple fading channel is implemented the BER efficiency [12].

According to several AS algorithms, TAS can minimize the BER but the code gain cannot be generated by this method. The advantages of code gain can be gained with upgrading BER by joining the TAS system in external code. From some existing work, integrated STBC and FEC codes gives greater code benefit with improved BER for MIMO systems [13-15]. The Low Density Parity Check (LDPC) codes incorporate with STBC improves the code gain by 9dB and the turbo code gives very high coding gain along with some other channel coding techniques [16]. In order to improve the performance of the space time coding, various combination techniques are proposed in some existing works. A novel hybrid STBC scheme is proposed in [17] which give increased performance compared to STBC without joint. The joint of STBC and TAS incorporated with TC obtained more than $4 \mathrm{~dB}$ SNR gain values [18]. The common selection combining technique used in cognitive radio is proposed [19].

In this paper, to prove the combinations of joint efficiency, the TAS-OSTBC system is the joint with several FEC codes in the initial process. TC utilized here as they perform near Shannon limit and the Turbo codes incorporated with $\mathrm{BCH}$ codes gives an improvement in coding gain [20]. Therefore, the TAS-OSTBC combination is proposed here with the internal TC and the external BCH code. The efficiency advantage of the TAS-OSTBC system with the enhanced code gain providing by this combination. The system analysis performance is performed on the Raleigh fading channel. By using multiple antenna systems all advantages of multiple antenna systems are obtained with complexity of less, improved error rates and code gain.

The main contribution of this paper is given below,

- To obtain the code gain high error correction with entire capacity advantages the TAS-OSTBC system proposed with joint $\mathrm{BCH}-\mathrm{Turbo}$ codes.

- The implementation of TAS-OSTBC system analysis is performed under Rayleigh fading.

- The Transmit Antenna Selection (TAS) system efficiency is analyzed first through joining along various FEC codes. From the analysis, the proposed Orthogonal Space Time Block Codes (OSTBC) -Turbo provides bit error rate that is around $10^{-5}$ for SINR of $9 \mathrm{~dB}$ but the similar for TAS without Forward Error Correction (FEC) would achieved over $15 \mathrm{~dB}$. Hence the inner joint is implemented through the TURBO codes.

- This proposed techniques performed better when compared to the OSTBC-LDPC concatenation.

The following sections of the paper are organized as: The preliminaries are discussed in Section 2 and in Section 3 the survey of the recent work related to the contribution is described. The TAS-OSTBC technique with the BCH-TURBO Code is explained in Section 4. In Section 5 the experimental results and discussions are reported and finally, the work is concluded in Section 6.

\section{Preliminaries}

\subsection{TAS-OSTBC System Joint with Various Coding schemes}

In low decoding problems for multiple transmit antenna systems the STBC provides essential benefits. Space-time codes joints with some FEC coding schemes such as TBCH (Turbo BCH codes), CC (Convolutional Codes), TCM (Trellis Coded Modulation), Turbo Convolutional codes and TTCM (Turbo Trellis Coded Modulation) for obtaining a large code advantages in some existing researches [21]. 


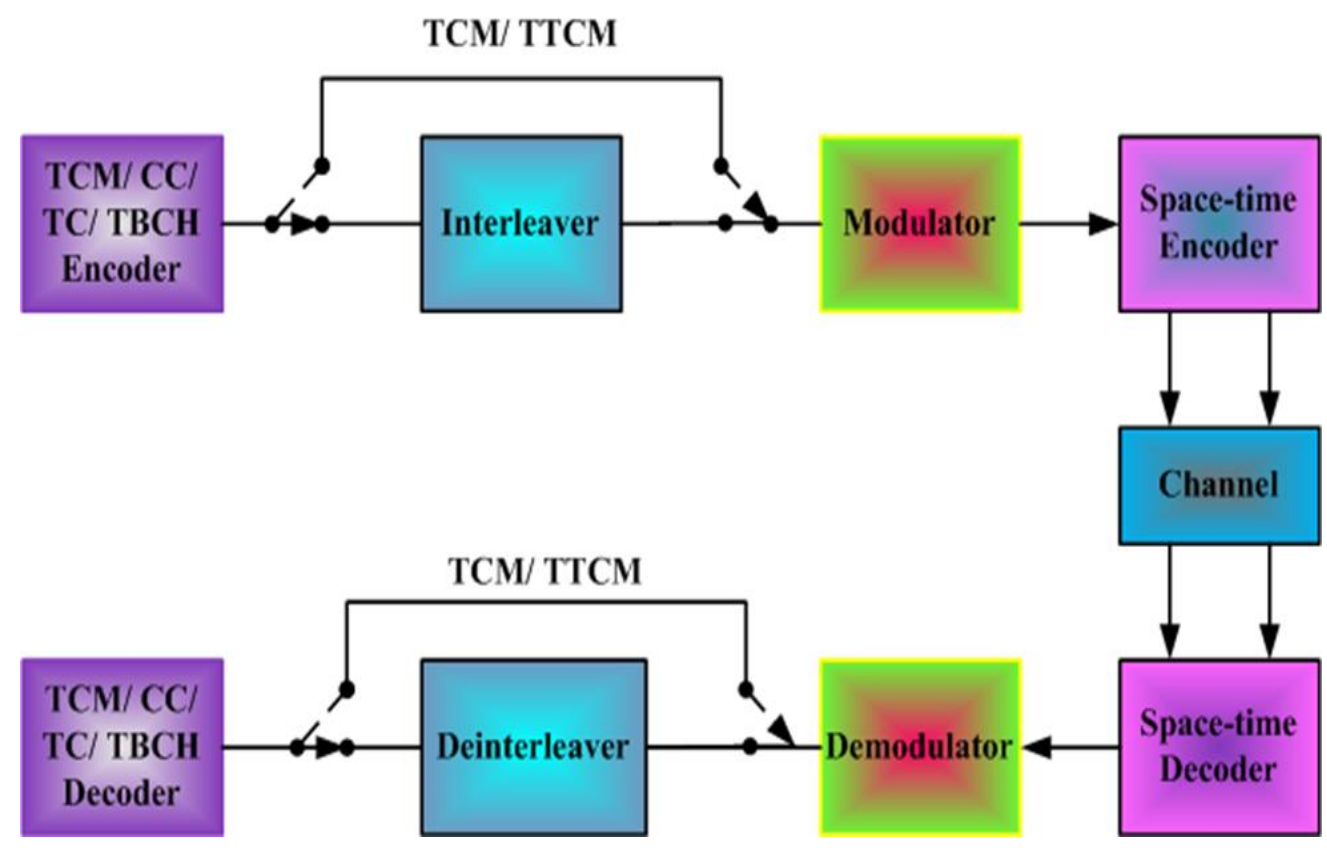

Fig. 1 - Systematic Structure of STBC with Various Coding Schemes

The MIMO-OSTBC utilizes multiple antennas in the transmitter and receiver and obtain high data rate as well as diversity gain [22]. In the transmitter and receiver having $N_{T}, N_{R}$ number of transmitting antennas respectively. Thus, $\left(N_{T}, N_{R}\right)$ is denoted as the Multiple Input Multiple Output system without the antenna selection. The controller sort out $L_{T}$ number of antennas out of $N_{T}$ antennas for transmission from the receiver based on the estimated CSI received. For the receiving antenna selection, it chooses the best $L_{R}$ antennas out of $N_{R}$ based on received SNR. If examine the TAS system which selects $L_{R}$ antennas, then it is known as $N_{T}: L_{R}, N_{R}$ system. The conventional MIMO-OSTBC system adopted the TAS technique and joint by FEC is shown in Figure 2 .

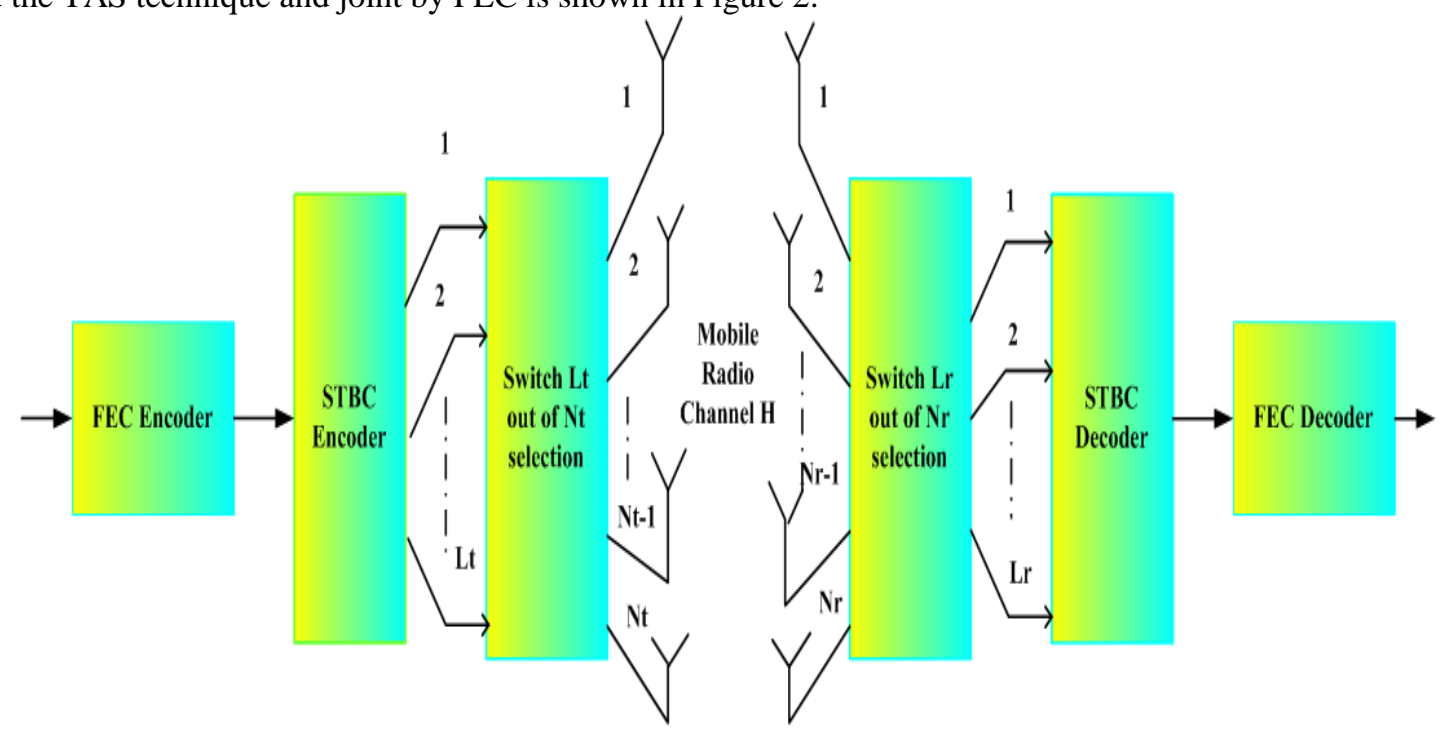

Fig. 2 - TAS based MIMO system with joint STBC with FEC

The size of the channel array consists of $\left(L_{T} \times L_{R}\right)$ here, $1 \leq i \leq L_{T}, 1 \leq j \leq L_{R}$ and $k_{i, j}$ are channel coefficients and the channel array represented as ${ }^{K}$.Examples of Rayleigh fading function are these fading coefficients. Then the Rayleigh distribution is followed by amplitude $\left|k_{i, j}\right|$. If noise vector $m$ and signal vector $a$ encoded by OSTBC, at the receiver side then the received signal input are consider by the following equation,

$\mathrm{b}=\mathrm{k} * \mathrm{a}+\mathrm{m}$ 
A Pilot-based training method is used to evaluate the SNR obtained. The Maximal Ratio Combining (MRC) method is utilized on the receiver to find the subset index with greater SNR. This subset index of the single selected TA indicated as ' $S$ ' the following equation is,

$$
\mathrm{S}=\arg \max _{1 \leq i \leq L}\left\{D_{i}=\sum_{j-1}^{L_{r}}\left|k_{i, j}\right|\right\}
$$

Let us assumed that the channel for data transmission is static and within the module this constant exist changes separately. The optimum values of BER are provided by the TAS system, where the OSTBC provides all diversity benefits with less hardware complexity. It is important to joint OSTBC system with few external codes to maximize code gain. FEC codes like convolutional code and block code performs the concatenation operation. In serial mode the one or very little bits of data are encoding by the Convolutional codes, whereas the block codes encoding the block of data. Various FEC codes such as Convolution Code (CC), Reed Solomon (RS), BCH, Trellis Coded Modulation (TCM) and Turbo Code are utilized here for verifying the performance of the TAS system.

The Multiple Input Multiple Output (MIMO) system efficiency improves with joint of convolutional codes as given in [23]. The combination of coding and modulation is named as the TCM system proposed by the Ungerboeck to improve the code gain. It contains the Trellis Code and the Constellation Mapper and the combination of coding and modulation decreases the Euclidian distance among the constellations. This provides the advantages of code gain due to the trellis code and overcome the power drawback of higher constellations.

Space-time trellis coding is scheming modulation, channel coding, variance of transmit and variance of optional receiver. By space-time trellis codes the operation performed is much better in more complex costs. MIMO-STBC joint with various FEC code improves the channel performance. The connection of two or more convolutional codes in parallel generated the Turbo code. It has two Recursive Systematic Convolution (RSC) codes. The bits of data are provided to the first RSC and the output is given to the second RSC is depicted in Fig 3.

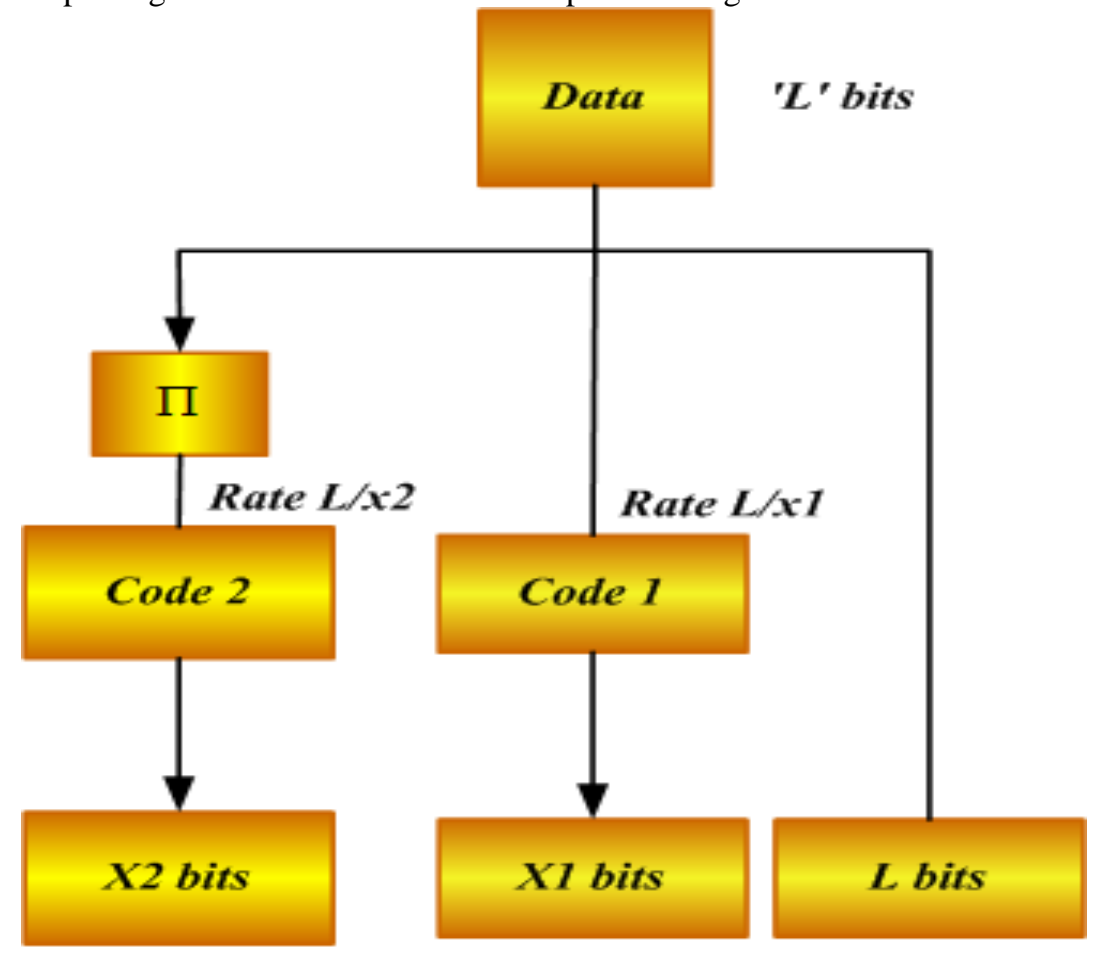

Global Code Rate $\mathrm{L} /(\mathrm{L}+\mathrm{x} 1+\mathrm{x} 2)$

Fig 3. Generalized structure of TURBO Encoder

The resulting code word has systematic bits $h(i)$, and two parity check streams $x_{1}(i), x_{2}(i)$ where $i=1,2, . . h$. The turbo decoder has two joint decoders as shown in Figure 4. 


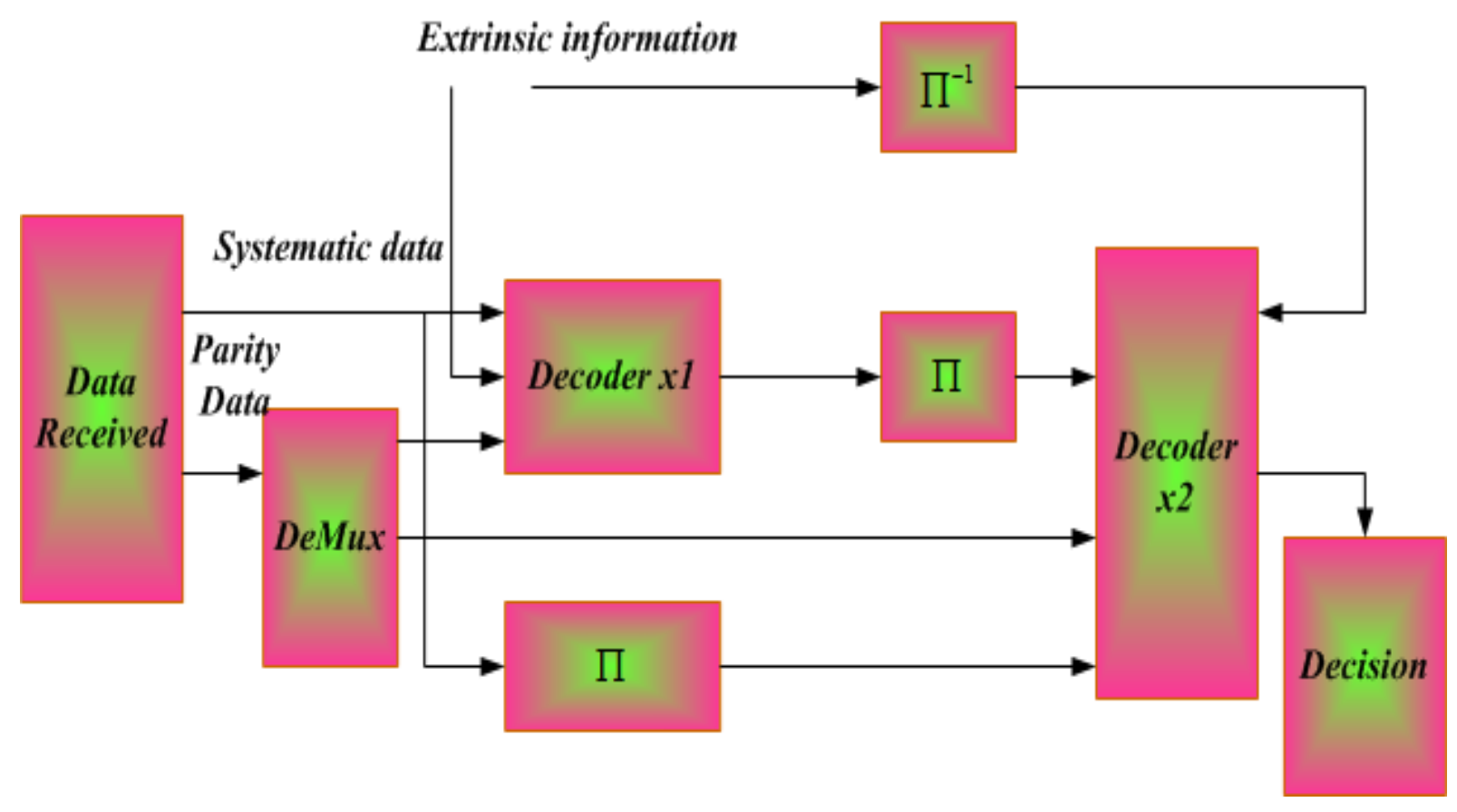

Fig. 4 - Generalized structure of TURBO decoder

In the first Decoder $x_{1}$ the systematic data and extrinsic information is provided. The result of the Decoder is a soft output and this soft output is a posteriori probabilities (APP) [24]. The second Decoder $x_{2}$ will process the similar data to examine the APP and it utilizes the BCJR algorithm for each iteration [25].

\section{Literature Survey}

\begin{tabular}{|c|c|c|c|c|}
\hline S.No & Authors & Methodology & Advantages & Disadvantages \\
\hline 1 & Chen, et al [26], 2019 & $\begin{array}{l}\text { For the antenna selection issues in the } \\
\text { massive MIMO system a self-supervised } \\
\text { learning based Monte Carlo Tree Search } \\
\text { (MCTS) method was proposed. The } \\
\text { complexity of search is reduced by a } \\
\text { regression module of linear and extracted } \\
\text { the features of channel from CSI } \\
\text { (Channel State Information). }\end{array}$ & $\begin{array}{l}\text { The searching complexity } \\
\text { was reduced. }\end{array}$ & $\begin{array}{l}\text { The hardware complexity } \\
\text { was high in this massive } \\
\text { MIMO antenna selection. }\end{array}$ \\
\hline 2 & $\begin{array}{l}\text { Damrath, et al [27], } \\
2018\end{array}$ & $\begin{array}{l}\text { Spatial transmission techniques in } \\
\text { multiple-input multiple output (MIMO) } \\
\text { system diffusion-based molecular } \\
\text { communications (DBMC) were } \\
\text { introduced for improved bit error rate. }\end{array}$ & $\begin{array}{l}\text { It was easy to access and } \\
\text { the time for computation } \\
\text { is less. }\end{array}$ & $\begin{array}{l}\text { The performance was not } \\
\text { better using this method } \\
\text { there was no code gain. }\end{array}$ \\
\hline 3 & $\begin{array}{l}\text { Eskandari, et al [28], } \\
2018\end{array}$ & $\begin{array}{l}\text { A novel antenna selection algorithm have } \\
\text { utilized for improved Energy Efficiency } \\
\text { (EE) and allocation of power issue and in } \\
\text { MIMO spatial multiplexing schemes, } \\
\text { there sends antenna's optimum subset } \\
\text { hint and power return level to the } \\
\text { transmitter through receiver. }\end{array}$ & $\begin{array}{l}\text { Power loading and the } \\
\text { complexity was } \\
\text { decreased. }\end{array}$ & \\
\hline 4 & $\begin{array}{l}\text { Huang, Yu, et al [29], } \\
2019\end{array}$ & $\begin{array}{l}\text { Random antenna selection (RAS) along } \\
\text { fast radio-frequency switching the } \\
\text { deterministic antenna selection based } \\
\text { PSM (DASPSM) was developed to PSM } \\
\text { (pre-coding-aided spatial modulation) for } \\
\text { improving secrecy performance. }\end{array}$ & $\begin{array}{l}\text { The complexity of } \\
\text { hardware was reduced. }\end{array}$ & $\begin{array}{l}\text { Selection process of the } \\
\text { transmit antenna was } \\
\text { difficult. }\end{array}$ \\
\hline 5 & Jabire, et al[30], 2018 & $\begin{array}{l}\text { The Authors proposed a full wave } \\
\text { simulation and MIMO antenna } \\
\text { characteristic mode-based structure for } \\
\text { wireless local area network applications. }\end{array}$ & $\begin{array}{l}\text { It increased the isolation } \\
\text { among the two monopoles } \\
\text { and reduced the } \\
\text { electromagnetic } \\
\text { interference. }\end{array}$ & $\begin{array}{l}\text { The antenna selection was } \\
\text { poor and the convergence } \\
\text { speed was low due to } \\
\text { complex computation. }\end{array}$ \\
\hline 6 & $\begin{array}{l}\text { Ratnam, Vishnu V., et } \\
\text { al [31], } 2018\end{array}$ & $\begin{array}{l}\text { For better selection of antennas a variant } \\
\text { of hybrid beamforming called hybrid } \\
\text { beamforming with selection (HBwS) }\end{array}$ & $\begin{array}{l}\text { It reduced the hardware } \\
\text { cost of multi-user } \\
\text { Massive Multiple-Input- }\end{array}$ & \\
\hline
\end{tabular}




\begin{tabular}{|c|c|c|c|c|}
\hline & & $\begin{array}{l}\text { program was recommended for the better } \\
\text { selection of antennas and the switch bank } \\
\text { architecture was utilized for the number } \\
\text { of users and channel estimation overhead } \\
\text { the effective system performance. }\end{array}$ & $\begin{array}{l}\text { Multiple-Output systems } \\
\text { and user separability, } \\
\text { beamforming gain, as } \\
\text { well as hardware } \\
\text { complexity is low. }\end{array}$ & \\
\hline 7 & Yang et al [32], 2019 & $\begin{array}{l}\text { A novel MIMO transmission method } \\
\text { based on pattern recognition (PR) was } \\
\text { introduced. KNN (K-nearest neighbor } \\
\text { algorithm) and SVM (support vector } \\
\text { machine algorithm) are the two PR } \\
\text { algorithms utilized for low-complexity as } \\
\text { well as high-efficient TAS. }\end{array}$ & $\begin{array}{l}\text { It has the capability to } \\
\text { solve the transmit antenna } \\
\text { selection problem and the } \\
\text { redundancy was reduced. }\end{array}$ & $\begin{array}{l}\text { The convergence speed was } \\
\text { low and required large } \\
\text { dimensional space. }\end{array}$ \\
\hline 8 & Zilli, et al [33], 2019 & $\begin{array}{l}\text { An adaptive beamforming technique was } \\
\text { utilized to solve the problem of } \\
\text { beamforming for uplink reception and } \\
\text { joint antenna selection in mobile } \\
\text { communication network systems. }\end{array}$ & $\begin{array}{l}\text { It increased the number of } \\
\text { antennas and reduced the } \\
\text { computational as well as } \\
\text { the hardware complexity. }\end{array}$ & $\begin{array}{l}\text { Due to the coupling among } \\
\text { the antenna selection matrix } \\
\text { and beamforming } \\
\text { coefficients, the } \\
\text { implementation of this } \\
\text { method was difficult. }\end{array}$ \\
\hline
\end{tabular}

In 2019, a self-supervised learning based Monte Carlo Tree Search (MCTS) method was introduced by Chen, et al [26] for the solution of antenna selection issues in a massive multiple inputs multiple output system. The complexity of search was reduced and the features of the channel from CSI (channel state information) were extracted by a regression module of linear. In this massive MIMO antenna selection the complexity of searching was reduced and the hardware complexity was high.

Damrath, et al [27] in 2018 have been proposed a spatial transmission technique and diffusion-based molecular communications (DBMC) for an enhanced BER in MIMO system. It was easy to access and time computation less. The performance was not better using this method because there was no code gain generated.

Eskandari, et al [28], 2018 have been utilized a novel antenna selection algorithm for Energy Efficiency (EE) improvement and problem of power allocation in MIMO spatial multiplexing schemes, where the hint of the antennas optimum subset was send by the receiver and level of the power return to the transmitter. Power loading and the complexity was decreased.

In PSM (pre-coding-aided spatial modulation), Deterministic antenna selection based PSM (DASPSM) and random antenna selection (RAS) along fast radio-frequency switching have been proposed by Huang, Yu, et al [29] in 2019 for improving performance of secrecy. The complexity of architecture was reduced but selection process of the transmit antenna was difficult.

For wireless local area network applications, a full wave simulation and multiple-input-multiple-output (MIMO) antenna characteristic mode-based structure have been introduced by Jabire, et al [30] in 2018. This increased the isolation among two monopoles and reduced the electromagnetic interference. The antenna selection was poor and the convergence speed was low due to complex computation.

In 2018, Ratnam, Vishnu V., et al [31]have been proposed a variant of hybrid beamforming called hybrid beamforming with selection (HBwS) scheme for the better selection of antennas and the controller framework for several users and the overhead estimation of channel effective system performance. It reduced the cost of hardware of multi-user Massive MIMO systems and user separability, beamforming gain, as well as hardware complexity was low.

In 2019, a novel multiple-input multiple-output (MIMO) transmission method based on pattern recognition (PR) was developed by Yang et al [32] for the best searching solution. KNN (K-nearest neighbor) algorithm and SVM (support vector machine) algorithm are the two PR algorithms utilized for low-complexity as well as high-efficient TAS. It has the capability to solve the transmit antenna selection problem and the redundancy was reduced and low BER (Bit Error Rate). The speed of convergence was low and required large dimensional space.

Zilli, et al [33], 2019 introduced an adaptive beamforming technique was utilized in this paper for solving the problem of beamforming for uplink reception and joint antenna selection in mobile communication network systems. This increased the number of antennas growth and reduced the computational as well as the hardware complexity. Owing to the connection among the selection matrix of the antenna and the beamforming coefficients, the implementation of this method was difficult.

\section{Proposed Joint of TAS-OSTBC System with BCH-TURBO Code}

As described in section 2, turbo codes are utilized in this paper because of their exceptional performance. The Turbo codes give improved performance as compared to LDPC codes [34]. The bit error rate is improved when the OSTBC system integrates with Turbo codes. The turbo code efficiency is increased by the outer BCH code by reduces the error base with rectification in the remaining errors. It increases the effects caused due to iterative APP Decoder and corresponding BER. Therefore, the combination of the BCH-TURBO code is considered in the proposed TAS-OSTBC system represents in fig 5 . 


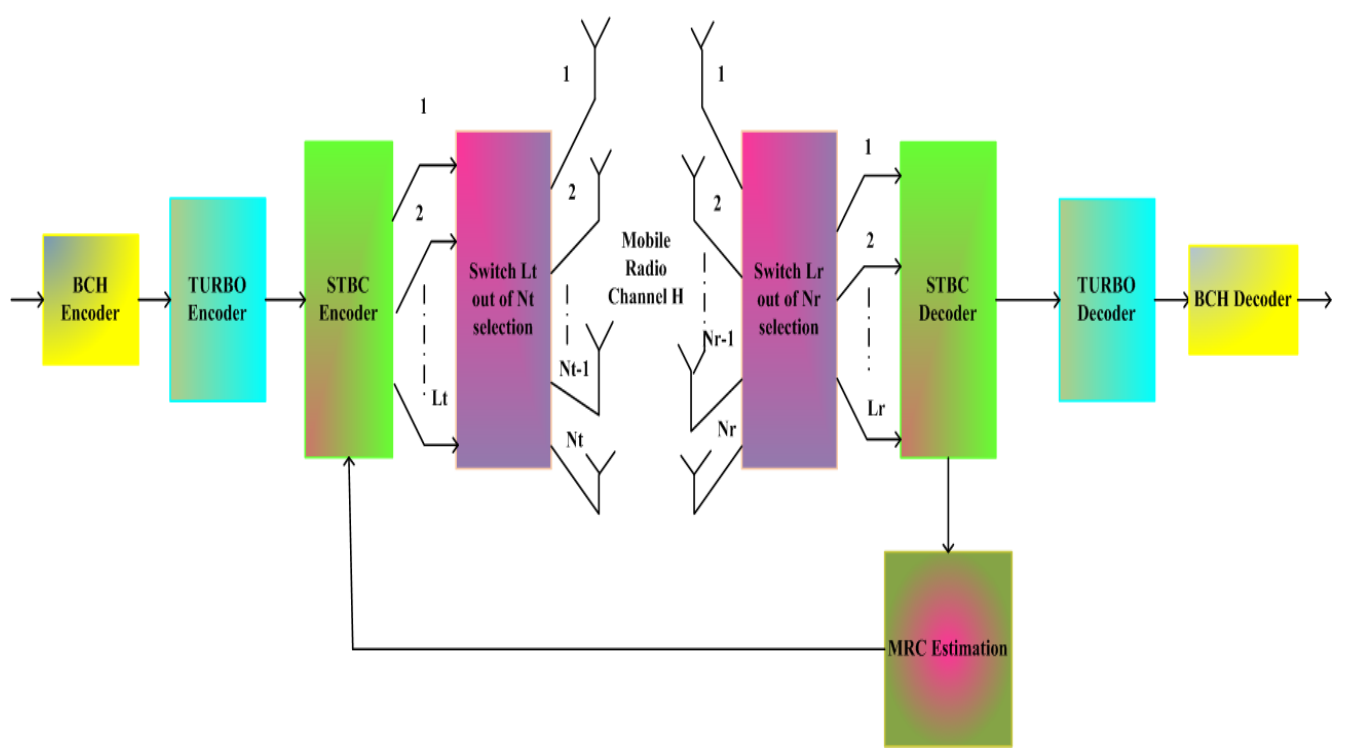

Fig 5. - Block diagram of proposed TAS-OSTBC with joint BCH-TURBO Code block diagram

The first data set is encoded using the external $\mathrm{BCH}$ encoder. Further the $\mathrm{BCH}$ code words encodes through inner TURBO code. The Turbo encoder processes the message with the packet length ${ }^{H}$ and generates the code word with length ${ }^{l}$. OSTB

$\mathrm{C}$ is used to encode the code words generated by the Turbo Code using the matrix $N$. The pilot based method is utilized to send pilots from $N_{T}$ transmitting antennas in each frame. In receiver for every pilot symbol, the corresponding received SNR is approximated.

The index IN of antenna subset along maximum value of received SNR is feedback to the transmitter as given in Eqn. (2). For transmission, the switch at the transmitter side will choose the best possible antenna based upon this feedback. Channel is considered flat blur i.i.d. channel whose arrival are i.i.d. with variance one zero mean circular random variable of Gaussian. The best state of channel data is accessible in receiver. Therefore the selection of ${ }_{T}$ out $N_{T}$ antennas becomes simple for transmission $L_{R}$ out of $N_{R}$ antennas in receiver.

As proposed [35] in terms of selection of suboptimal several AS is observed. According to the equation (3) the Maximal Ratio Combining (MRC) maximum value were given by the antenna's first subset that is chosen. In descending order arrangement, the antenna's subset indices evaluated through Maximal Ratio Combining (MRC). ${ }_{T}$ represents as the number of antenna selected for transmission and $D(1)$ are the indices arranged as $D(1) \leq D(2) \leq \ldots . \leq D\left(L_{T}\right)$ in descending order. The instantaneous SNR $\lambda\left(L_{T}\right)$ provides $\lambda\left(L_{T}\right)=D\left(L_{T}\right) \bar{\lambda}$ for single TAS/MRC case (single Transmit

Antenna Selection/Maximal Ratio Combining). $Q_{x}\left(D_{i} \rightarrow D_{j} \mid K_{\left\{Q_{1}, Q_{2}, \ldots Q_{P}\right\}}\right)$. Here the channel is considered as
Pair wise probability error is indicated by $K_{\left\{Q_{1}, Q_{2}, \ldots Q_{P}\right\}}, j \neq i$. To the pair-wise error probability the upper limit is expressed as,

$$
\begin{gathered}
\mathrm{Q}_{\mathrm{x}}\left(D_{i} \rightarrow D_{j} \mid K_{\left\{Q_{1}, Q_{2, \ldots}, Q_{P}\right\}}\right)=P\left(\sqrt{\frac{\rho\left\|K_{\left\{Q_{1}, Q_{2, \ldots}, Q_{P}\right\}} G_{i, j}\right\|^{2} H}{2 N_{T}}}\right) \\
Q_{x}\left(D_{i} \rightarrow D_{j} \mid K_{\left\{Q_{1}, Q_{2, \ldots,}, Q_{P}\right\}}\right) \leq \exp \left(-\frac{\rho\left\|K_{\left\{Q_{1,}, Q_{2, \ldots}, Q_{P}\right\}} G_{i, j}\right\|^{2} H}{4 N_{T}}\right)
\end{gathered}
$$

According to Equation (3) the number of chosen antenna subset reduces the upper limit is represented as $P$ and expressed as

$$
\left\{Q_{1}^{O P T}, Q_{2}^{O P T}, \ldots ., Q_{P}^{O P T}\right\}=\underset{Q_{1}, Q_{2} \ldots, Q_{P} \in X_{P}}{\arg \max }\left\|K_{\left\{Q_{1}, Q_{2, \ldots}, Q_{P}\right\}} G_{i, j}\right\|^{2} H
$$




$$
\left\{Q_{1}^{O P T}, Q_{2}^{O P T}, \ldots, Q_{P}^{O P T}\right\}=\underset{Q_{1}, Q_{2} \ldots, Q_{P} \in X_{P}}{\arg \max } \| K_{\left\{Q_{1}, Q_{2}, \ldots, Q_{P}\right\}} G_{i, j} G_{i, j}{ }^{R} R_{\left\{Q_{1}, Q_{2} \ldots, Q_{P}\right\}}^{R}
$$

Moreover, with $P$ chosen antennas of $\left\{Q_{i}\right\}_{i=1}^{P}$, the average SNR is represented as

$$
\eta\left\{Q_{1, Q_{2}, \ldots,} Q_{P}\right\}=\frac{\rho}{P}\left\|K_{\left\{Q_{1}, Q_{2, \ldots,}, Q_{P}\right\}} G_{i, j}\right\|^{2} H
$$

Based on eqn (6) and (7) the antenna subset is chosen. The received average SNR then the selection of optimal $P$ antennas is given as

$$
\frac{\rho}{\mathbf{P}}\|K\|^{2} H \geq \eta_{\left\{Q_{1}, Q_{2}, \ldots, Q_{P}\right\}} \geq \frac{\rho}{N_{T}}
$$

The pair wise error probability is measured by taking inner space time turbo code, eqn (4) is rearranged by applying Chern-off bounds and substituting the values of $K^{2}$ as given in

$$
Q_{x}\left(D_{1} \rightarrow D_{2} \mid K\right) \leq \frac{1}{2} \prod_{t=1}^{1} e^{\left(\frac{-\rho L_{R}}{4 N_{T} N_{R}} \sum_{j=1}^{\left.N_{R} \sum_{i=1} k_{t, i, j}^{2}\left(v_{1, t}-v_{2, t}\right)^{2}+1\right)}\right.}
$$

where $\rho=\frac{G_{s}}{N_{o}}$, Substituting $\frac{-\rho L_{R}}{4 N_{T} N_{R}}\left(v_{1, t}-v_{2, t}\right)^{2}=\tau_{\pi}^{2}$ and $\sum_{j=1}^{N_{R}} \sum_{i=1}^{N_{T}} k_{t, i, j}^{2}=Y_{t}$. Also, consider that $Y_{t}$ is a random variable and have square distribution of chi each along $2 N_{T} N_{R}$ freedom degree. By considering the PDF of Rayleigh fading channel as given below,

$$
J_{Z}\left(w_{T}\right)=\frac{1}{\left(N_{T} N_{R}-1\right) !} e^{-W_{t}} W_{t}^{\left(N_{T} N_{R}-1\right)}
$$

Substituting Eqn (10) into (9) and solving.

$$
\begin{aligned}
& Q_{x}\left(D_{i} \rightarrow D_{j}\right) \leq \int_{0}^{\infty} \frac{1}{2} \prod_{t=1}^{1} e^{\left(\tau_{t}^{2}\right)} \frac{1}{\left(N_{T} N_{R}-1\right) !} e^{-W_{t}} W_{t}^{\left(N_{T} N_{R}-1\right)} D w_{t} \\
& Q_{x}\left(D_{i} \rightarrow D_{j}\right) \leq \frac{1}{2\left(N_{T} N_{R}-1\right) !} \prod_{t=1}^{1} \int_{0}^{\infty} e^{-W_{t}} W_{t}^{\left(N_{T} N_{R}-1\right)} D w_{t}
\end{aligned}
$$

Substituting $W_{t}\left(\tau_{\tau}^{2}+1\right)=Z_{t}$ and solving, the probability of pair wise error for TAS system is represented as,

$$
Q^{T A S}=Q_{r}\left(D_{i} \rightarrow D_{j}\right) \leq \frac{1}{2} \prod_{t=1}^{1}\left(\frac{-\rho L_{R}}{4 N_{T} N_{R}}\left(v_{1, t}-v_{2, t}\right)^{2}+1\right)^{-N_{T} N_{R}}
$$

By assuming $D=[D[1], D[2], \ldots D[C]$ are independent, the total pair wise error probability of inner TURBO code with antenna selection is represented as,

$$
Q\left(D \rightarrow D^{\prime}\right)=\prod_{c=1}^{C} Q^{T A S}\left(D_{1, c} \rightarrow D_{2, c}\right)
$$

Additionally, all $C$ received code vectors are assembled together; the pair wise error probability of the outer BCH code with antenna selection is expressed as 


$$
Q\left(d \rightarrow d^{\prime}\right) \leq Q\left(D \rightarrow D^{\prime}\right)=\frac{1}{2} \prod_{c=1}^{C}\left(\frac{\rho L_{R}}{4 N_{T} N_{R}}\left(\mid\left(D_{1, c} \rightarrow v_{2, c}\right)^{2}+1\right)^{-N_{T} N_{R}}\right)
$$

If the eqn (15) is compared with normal conventional MIMO system without antenna selection, it is studied that the code gain loss is upper limited by $10 \log _{10} \frac{N_{R}}{L_{R}} d B$.

\section{Experimental Results and Discussion}

The TAS-OSTBC system efficiency analysis implemented by the Rayleigh fading channel and proved at initial for the Forward Error Correction codes. In section 2, the TAS-OSTBC system efficiency combination is discussed with generalized FEC codes. TURBO, CC, BCH, RS, TCM are the various FEC coding methods taken for analysis. The Transmit Antenna Selection (TAS) system efficiency is analyzed by comparing with and without combining of Forward Error Correction codes with OSTBC. The parameters for the performance analysis are given in below table 1 .

Table 1 - The performance analysis parameters

\begin{tabular}{ccc}
\hline S.No & Parameters & Value \\
\hline 1 & BCH Message Length (k) & 04 \\
2 & BCH codeword Length (n) & 13 \\
3 & TURBO code Block Length & 12000 \\
4 & BCH Code Rate & 0.33 \\
5 & Convolution Code Rate & 0.5 \\
\hline
\end{tabular}

Table 1 shows the performance analysis parameters. The value for $\mathrm{BCH}$ message length is 04 , $\mathrm{BCH}$ code word length is 13, TURBO code Block Length value is 12000 , BCH Code Rate value is 0.33 and Convolution Code Rate is 0.5 .

Figure 6 represented the performance of the OSTBC-Turbo code with various coding techniques and result of the OSTBC-Turbo code is best to compare other methods. The OSTBC-Turbo provides bit error rate that is nearly $10^{-6}$ to SINR (signal-to-interference-plus-noise ratio) of $9 \mathrm{~dB}$. At the same time, similar would achieve over $15 \mathrm{~dB}$ for Transmit Antenna Selection (TAS) without Forward Error Correction (FEC). Hence, OSTBC are utilized as inner code joint along with Turbo codes for the proposed method.

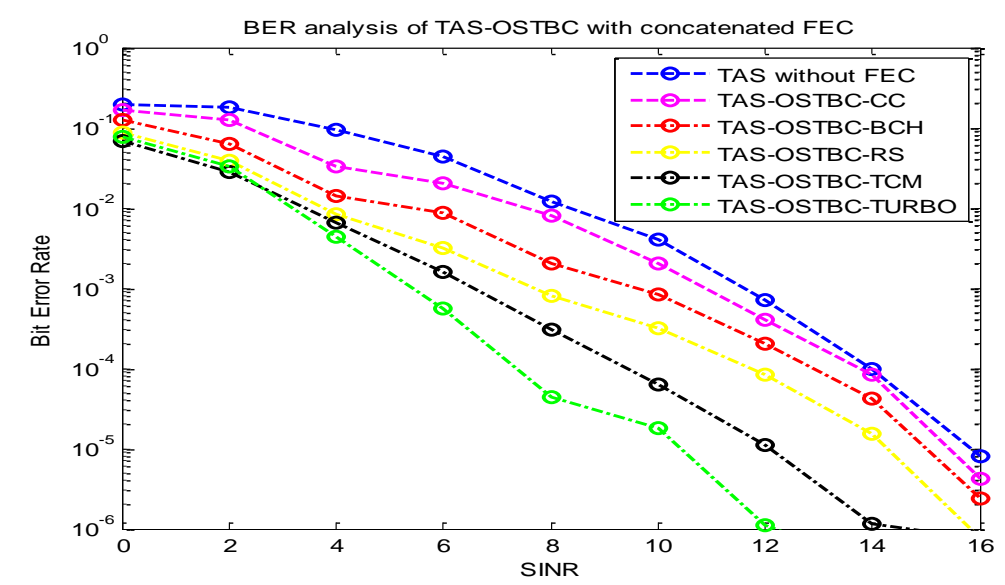

Fig 6. Bit error rate evaluation of TAS-OSTBC (Transmit Antenna Selection-Orthogonal Space Time Block Codes) with joint FEC 


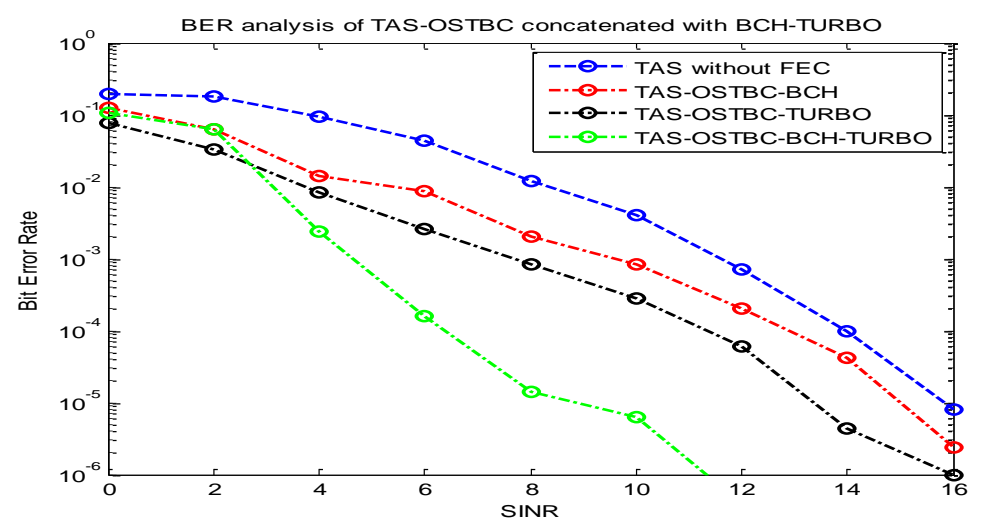

Fig 7. BER evaluation of TAS-OSTBC (Transmit Antenna Selection-Orthogonal Space Time Block Codes)with joint Bose-Chaudhuri-Hocquenghem (BCH)-Turbo Code

BER(Bit Error Rate) analysis implemented by the Rayleigh fading channel to the presented TAS-OSTBC joint along Bose-Chaudhuri-Hocquenghem-Turbo code as shown in above Figure 7. In view of TAS-OSTBC (Transmit Antenna Selection-Orthogonal Space Time Block Codes)-Bose-Chaudhuri-Hocquenghem (BCH) gives Bit Error Rate (BER) at the $10^{-5}$ limit for SINR of $7 \mathrm{~dB}$ at similar time obtained to greater than $14 \mathrm{~dB}$ to joint Transmit Antenna SelectionOrthogonal Space Time Block Codes-Turbo code. From figure 7, TAS without FEC achieves the highest value compared to the remaining three curves. TAS-OSTBC- BCH-TURBO attains lowest value in this figure.

The TAS-OSTBC system provided in the Bose-Chaudhuri-Hocquenghem (BCH)-Turbo code shows better than the combined TAS-OSTBC system in combination with the Turbo code and the Transmit Antenna Selection-Orthogonal Space Time Block Codes (TAS-OSTBC) system combined with the LDPC code. From Figure 8 perceived that BER (Bit Error Rate) of $10^{-4}$ is obtained in $7 \mathrm{~dB}$ at presented system when compared to Orthogonal Space Time Block Codes (OSTBC)-LDPC system which obtained in $11 \mathrm{~dB}$.

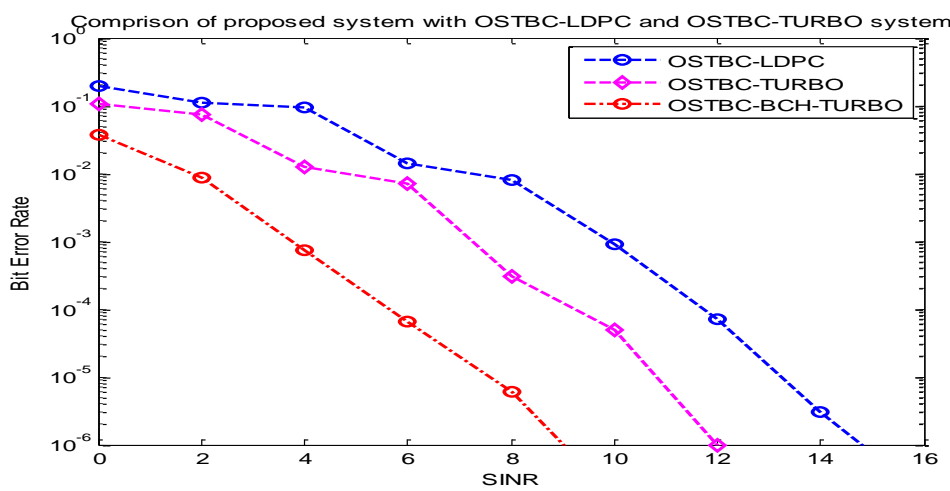

Fig 8. Comparison of performance analysis of TAS-OSTBC analysis with various systems

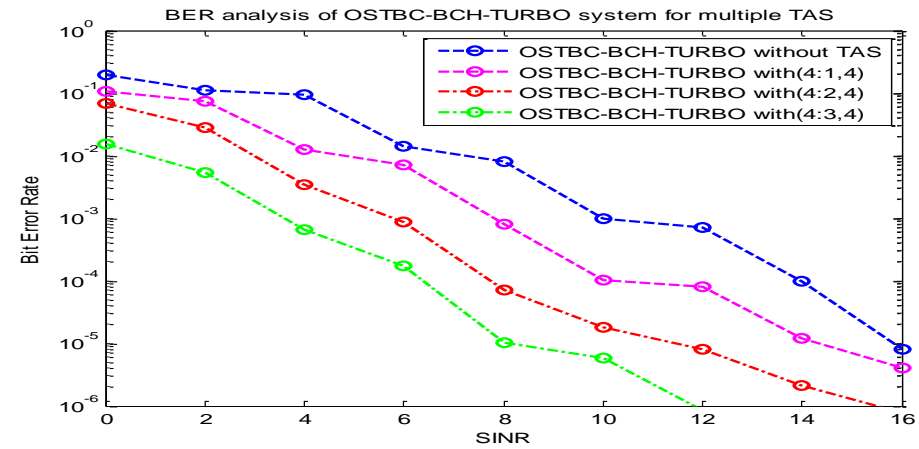

Fig 9.OSTBC-BCH-TURBO systems bit error rate analysis for several TAS

The bit error rate evaluation of the proposed method is implemented for multiple Transmit Antenna Selection technique is depicted in Figure 9. The conventional MIMO system refers to (4x4) MIMO (Multi Input Multi Output) without TAS (Transmit Antenna Selection). Hence, from (4x4) MIMO the single Transmit Antenna Selection (TAS) 
system is denoted as (4:1.4). Finally, from the four transmit antennas the $(4: 3,4)$ and $(4: 2,4)$ system represents three and two antenna selection respectively. The bit error rate evaluation comparison for $4 \times 4$ MIMO with and without FEC is done for verifying the system performance. Therefore, the comparison for single and double antenna selection with and without Forward Error Correction (FEC) with TAS (Transmit Antenna Selection) case is done. Comparatively, the performance of the system is improved compared to that of the system $(4,1.4)$ by selecting two transmit antennas $(4,2$. 4). The number of selected subset transmitting antennas increasing along with the system efficiency improves as shown in the above figures.

Table 2: Comparison of the performance of the proposed method with the existing method

\begin{tabular}{|c|c|c|}
\hline Performance & Proposed Method & Existing [35] \\
\hline $\begin{array}{l}\text { Bit error rate evaluation of TAS- } \\
\text { OSTBC with joint FEC }\end{array}$ & $10^{-2}$ & $10^{-1}$ \\
\hline $\begin{array}{l}\text { BER evaluation of TAS-OSTBC } \\
\text { with BCH Turbo Code }\end{array}$ & $10^{-2.5}$ & $10^{-2}$ \\
\hline $\begin{array}{l}\text { OSTBC-BCH-TURBO systems bit } \\
\text { error rate analysis for several TAS }\end{array}$ & $10^{-2}$ & $10^{-1}$ \\
\hline
\end{tabular}

\section{Conclusion}

In this paper to obtain the code gain high error correction with entire capacity advantages the TAS-OSTBC system proposed with joint BCH-Turbo codes. The implementation of the TAS-OSTBC system analysis is performed under Rayleigh fading. The Transmit Antenna Selection (TAS) system efficiency is first analyzed by combining various FEC codes. From the analysis, the proposed Orthogonal Space Time Block Codes (OSTBC) -Turbo provides bit error rate that is around $10^{-5}$ for SINR of $9 \mathrm{~dB}$ but more than $15 \mathrm{~dB}$ for a TAS without Forward Error Correction (FEC). Hence the inner joint is implemented through the TURBO codes. In addition, the outer joint is implemented using the Bose-ChaudhuriHocquenghem $(\mathrm{BCH})$ code to increase the code gain. From the simulation results, the proposed method provides an improvement of $4 \mathrm{~dB}$ at $10^{-6}$ with OSTBC-LDPC concatenation when compared to the Turbo code combination and the proposed method performance also analyzed. This proposed technique is performed better compared to the OSTBCLDPC concatenation. The performance of the TAS-OSTBC techniques is improved through the number of subsets that transmitting antennas with reduced hardware complexity, which provides the advantages of increased capacity with coding gain. In future work will expand the scope of our research into multi-cellular scenarios.

\section{Reference}

[1] Shaik, P., Singya, P., \& Bhatia, V. (2019). Performance analysis of QAM schemes for non-regenerative cooperative MIMO network with transmit antenna selection. AEU - International Journal Of Electronics And Communications, 107, 298-306. doi: 10.1016/j.aeue.2019.05.012

[2]Lan, S., Yang, C., \& Chen, C. (2019). Online Car-Hailing System Performance Analysis Based on Bayesian Network. IEEE Access, 7, 101195-101212. doi: 10.1109/access.2019.2929620

[3] Zhu, L., Zhu, J., Wang, S., \& Zhang, J. (2019). Adaptive Transmit Antenna Selection Based on PCA for Millimeter Wave LOS MIMO Channel. IEEE Access, 7, 12087-12096. doi: 10.1109/access.2019.2892869

[4] Selvam, P., \& Vishvaksenan, K. (2019). Antenna Selection and Power Allocation in Massive MIMO. Radioengineering, 27(1), 340-346. doi: 10.13164/re.2019.0340

[5] Molisch, A., \& Win, M. (2004). MIMO systems with antenna selection. IEEE Microwave Magazine, 5(1), 46-56. doi: 10.1109/mmw.2004.1284943

[6] Rao, I., \& Malleswara Rao, V. (2019). Optimal Transmit Antenna Selection Using Improved GSA in Massive MIMO Technology. Wireless Personal Communications, 109(2), 1217-1235. doi: 10.1007/s11277-019-06611-8

[7] Le, T., \& Kong, H. (2019). Energy harvesting relay-antenna selection in cooperative MIMO/NOMA network over Rayleigh fading. Wireless Networks. doi: 10.1007/s11276-019-02051-1

[8] Jin, G., Zhao, C., Fan, Z., \& Jin, J. (2019). Antenna Selection in TDD Massive MIMO Systems. Mobile Networks And Applications. doi: 10.1007/s11036-019-01297-5

[9] Lee, D. (2019). Performance analysis of opportunistic beamforming system under non-identical imperfect channel environment. IET Communications, 13(11), 1586-1594. doi: 10.1049/iet-com.2018.6115

[10] Cai, Y., Zhao, M., Shi, Q., Champagne, B., \& Zhao, M. (2016). Joint Transceiver Design Algorithms for Multiuser MISO Relay Systems with Energy Harvesting. IEEE Transactions On Communications, 1-1. doi: 10.1109/tcomm.2016.2605688 
[11] Tang, H., Zong, X., \& Nie, Z. (2018). Global-Searching-Based Iterative Swapping Antenna Selection for Massive MIMO Systems with Imperfect Channel Estimation. IEEE Access, 6, 66557-66564. doi: 10.1109/access.2018.2878732

[12] Zhu, L., Zhu, J., Wang, S., \& Zhang, J. (2019). Adaptive Transmit Antenna Selection Based on PCA for Millimeter Wave LOS MIMO Channel. IEEE Access, 7, 12087-12096. doi: 10.1109/access.2019.2892869

[13] Fountoukidis, K., Kalialakis, C., Psannis, K., Siakavara, K., Goudos, S., Sarigiannidis, P., \& Obaidat, M. (2018). MIMO antenna selection using biogeography-based optimization with nonlinear migration models. International Journal Of Communication Systems, 31(17), e3813. doi: 10.1002/dac.3813

[14] Chen, N., Ma, J., Wang, M., \& Shen, X. (2018). Two-Tier Energy Compensation Framework Based on Mobile Vehicular Electric Storage. IEEE Transactions On Vehicular Technology,67(12), 11719-11732. doi: 10.1109/tvt.2018.2874046

[15] Payami, S., Ghoraishi, M., Dianati, M., \& Sellathurai, M. (2018). Hybrid Beamforming With a Reduced Number of Phase Shifters for Massive MIMO Systems. IEEE Transactions On Vehicular Technology, 67, 4843-4851. doi: 10.1109/tvt.2018.2807921

[16] Sarker, M., Lee, M., \& Chinnadurai, S. (2018). Sub-optimal Antenna Selection in the High SNR MIMO Correlated Downlink Channel. Wireless Personal Communications, 99(4), 1713-1724. doi: 10.1007/s11277-018-5339-8

[17] 2018 Index journal of communications and networks, volume 20. (2018). Journal Of Communications And Networks, 20, 601-605. doi: 10.1109/jen.2018.000092

[18] González, D., da Costa, D., \& Santos Filho, J. (2016). Distributed transmit-antenna selection in variable-gain relaying systems. Wireless Networks, 24(4), 1161-1174. doi: 10.1007/s11276-016-1395-8

[19] Issue Information - TOC. (2017). IEEJ Transactions On Electrical And Electronic Engineering, 13(1), 1-3. doi: $10.1002 /$ tee. 22535

[20] Attarkashani, A., Hamouda, W., Moualeu, J., \& Haghighat, J. (2019). Performance Analysis of Turbo Codes and Distributed Turbo Codes in Buffer-Aided Relay Systems. IEEE Transactions On Communications, 67(7), 46204633. doi: 10.1109/tcomm.2019.2911280

[21] Bee Leong Yeap, Tong Hooi Liew, Hamorsky, J., \& Hanzo, L. (2002). Comparative study of turbo equalization schemes using convolutional, convolutional turbo, and block-turbo codes. IEEE Transactions On Wireless Communications, 1(2), 266-273. doi: 10.1109/7693.994820

[22] Naima, S., Debbat, F., \& Bendimerad, F. (2018). Performance Improvement of MIMO-OSTBC System with BCHTURBO Code In Rayleigh Fading Channel. Indonesian Journal Of Electrical Engineering And Computer Science, 11, 898. doi: 10.11591/ijeecs.v11.i3.pp898-907

[23] Recent Analysis of Green Energy Technology (GET) for Telecom Applications. (2019). International Journal Of Recent Technology And Engineering, 8(4), 1326-1332. doi: 10.35940/ijrte.d7155.118419

[24] 2015 Index IEEE Transactions on Circuits and Systems I: Regular Papers Vol. 62. (2015). IEEE Transactions On Circuits And Systems I: Regular Papers, 62, 2953-2996. doi: 10.1109/tcsi.2015.2514200

[25] de Souza, I., Alcoforado, M., \& da Rocha, V. (2017). Turbo decoding of simple product codes in a two user binary adder channel employing the Bahl-Cocke-Jelinek-Raviv algorithm. Telecommunication Systems, 68(3), 513-521. doi: 10.1007/s11235-017-0407-z

[26] Chen, J., Chen, S., Qi, Y., \& Fu, S. (2019). Intelligent Massive MIMO Antenna Selection Using Monte Carlo Tree Search. IEEE Transactions On Signal Processing, 67(20), 5380-5390. doi: 10.1109/tsp.2019.2940128

[27] Damrath, M., Yilmaz, H., Chae, C., \& Hoeher, P. (2018). Array Gain Analysis in Molecular MIMO Communications. IEEE Access, 6, 61091-61102. doi: 10.1109/access.2018.2875925

[28] Eskandari, M., Doost Hoseini, A., \& Fazel, M. (2019). An energy-efficient joint antenna selection and power allocation for MIMO systems under limited feedback. Signal Processing, 163, 66-74. doi: 10.1016/j.sigpro.2019.05.010

[29] 2019 Index IEEE Transactions on Vehicular Technology Vol. 68. (2019). IEEE Transactions On Vehicular Technology, 68, 12453-12642. doi: 10.1109/tvt.2020.2968232

[30] Jabire, A., Zheng, H., Abdu, A., \& Song, Z. (2019). Characteristic Mode Analysis and Design of Wide Band MIMO Antenna Consisting of Metamaterial Unit Cell. Electronics, 8(1), 68. doi: 10.3390/electronics8010068

[31] 2018 Index IEEE Transactions on Signal Processing Vol. 66. (2018). IEEE Transactions On Signal Processing, 66, 6577-6638. doi: 10.1109/tsp.2018.2889430

[32] Yang, P., Zhu, J., Xiao, Y., \& Chen, Z. (2019). Antenna selection for MIMO system based on pattern recognition. Digital Communications And Networks, 5(1), 34-39. doi: 10.1016/j.dcan.2018.10.001

[33] Zilli, G., Pitz, C., Batista, E., Seara, R., \& Zhu, W. (2019). An Adaptive Approach for the Joint Antenna Selection and Beamforming Optimization. IEEE Access, 7, 99017-99030. doi: 10.1109/access.2019.2930029

[34] Hwang, I., Park, H., \& Lee, J. (2019). LDPC Coded Massive MIMO Systems. Entropy, 21, 231. doi: $10.3390 / \mathrm{e} 21030231$

[35] Hendre, V., Murugan, M., \& Chandgude, A. (2015). Multiple Transmit Antenna Selection in MIMO-OSTBC System in Nakagami-m Fading Channel. Procedia Computer Science, 54, 558-565. doi: 10.1016/j.procs.2015.06.064 